Supporting Information for:

\title{
Simultaneous selective-area and vapor-liquid-solid growth of InP nanowire arrays
}

Qian Gao, Vladimir G. Dubrovskii, Philippe Caroff, Jennifer Wong-Leung, Li Li, Yanan Guo, Lan Fu, Hark Hoe Tan, and Chennupati Jagadish

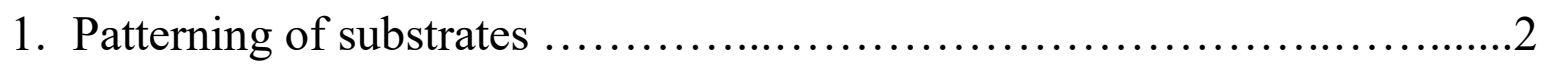

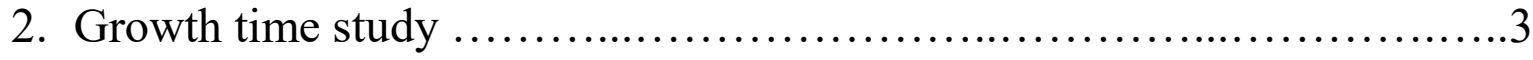

3. Cooling down under $\mathrm{AsH}_{3}$ protection instead of $\mathrm{PH}_{3} \ldots \ldots \ldots \ldots \ldots \ldots \ldots . . \ldots 4$

4. Nanowires grown on substrates patterned by FIB .....................5

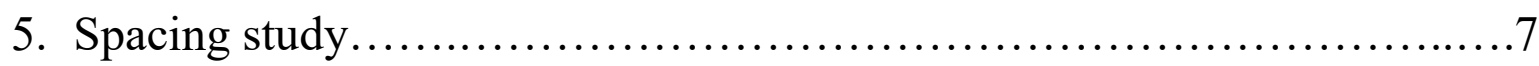

6. Model for diffusion flux and nanowire length.........................8

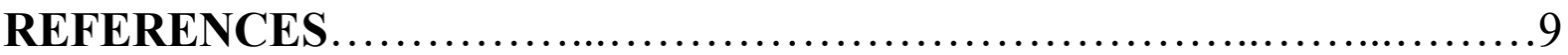




\section{Patterning of substrates}

A $30 \mathrm{~nm}-\mathrm{SiO}_{\mathrm{x}}$ layer was deposited on (111)A InP substrates by plasma-enhanced chemical vapor deposition (PECVD). Two methods are used to pattern the substrate: electron beam lithography (EBL) and focused ion beam (FIB).

\subsection{Electron beam lithography}

Hexamethyldisilazane (HMDS) was first spin-coated on the substrate to enhance the adhesion between the resist and substrate, followed by spin-coating of an electron beam resist (ZEP 520A resist). ${ }^{1}$ EBL system was used to pattern the resist with a hexagonal array of holes. After development of the resist, oxygen plasma was used to remove the footage residues of the resist prior to etching of the pattern using inductively coupled plasma reactive ion etching (ICP-RIE). The $\mathrm{SiO}_{x}$ on patterned area was etched by $\mathrm{CHF}_{3}$ gas $(40 \mathrm{sccm})$ at a rate of $1 \mathrm{~nm} / \mathrm{s}$ at $20{ }^{\circ} \mathrm{C}$ with a processing pressure of $10 \mathrm{mTorr}$, an $\mathrm{RF}$ power of $60 \mathrm{~W}$ and a ICP power of $400 \mathrm{~W}$. ZEP stripper was used to remove the resist and oxygen plasma was again used to clean the surface.

\subsection{Focused ion beam}

In some samples, FIB was also used to directly pattern the $\mathrm{SiO}_{\mathrm{x}}$-coated $\mathrm{InP}$ substrate. The voltage and current of the ion beam are $30 \mathrm{keV}$ and $93 \mathrm{pA}$ respectively for the milling process, and $8 \mathrm{keV}$ and $21 \mathrm{pA}$ respectively for the cleaning process.

Regardless of samples patterned by EBL/ICP-RIE or FIB, a trim etch procedure was carried out after patterning to remove the top thin layer of InP substrate which could have been damaged during ICP or gallium beam milling process. $10 \% \mathrm{H}_{2} \mathrm{O}_{2}$ was first used to oxidize the InP layer for $2 \mathrm{~min}$. Then a clean InP surface was prepared by etching off the 
oxide layer with $10 \% \mathrm{H}_{3} \mathrm{PO}_{4}$ solution for $2 \mathrm{~min}$. These steps were repeated sequentially for 5 times. Samples were then immediately transferred into the metalorganic vapor phase epitaxy (MOVPE) reactor for nanowire (NW) growth after the trim etching step.

\section{Growth time study}
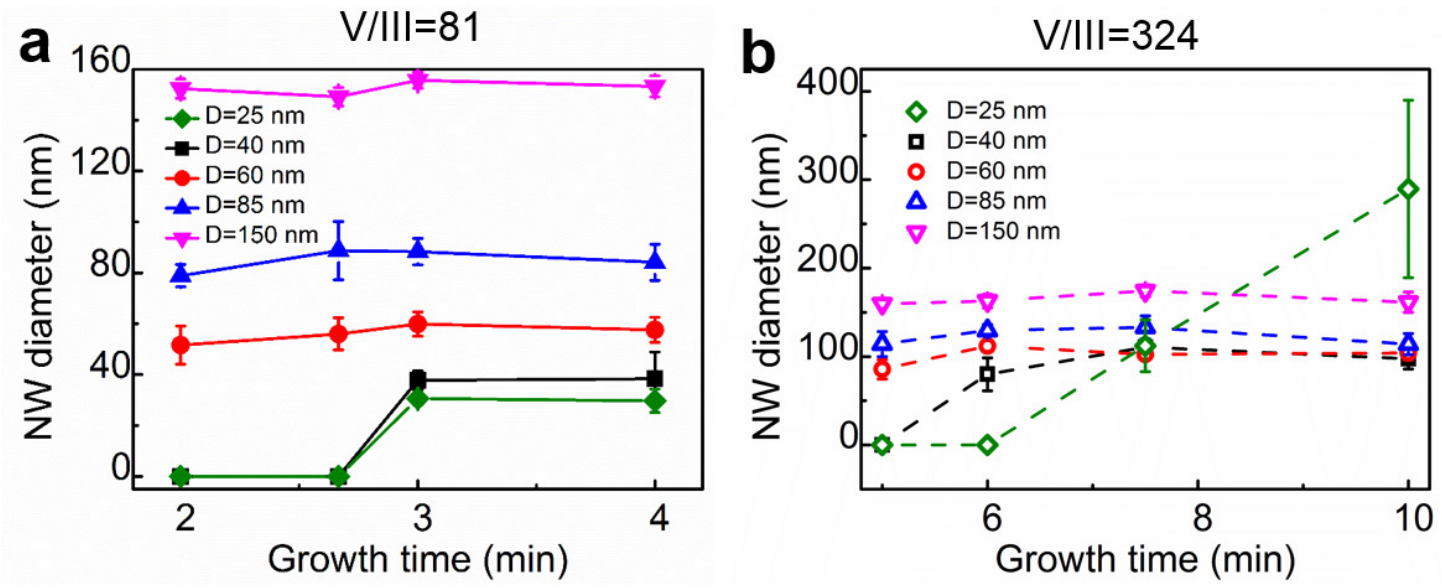

Figure S1. Graph showing the NW diameter as a function of growth time for the V/III ratios of (a) 81 and (b) 324. The NWs grown from the openings with a spacing of $500 \mathrm{~nm}$ and diameters of $25,40,60,85$ and $150 \mathrm{~nm}$ have been investigated. The solid and dashed lines are guide for the eyes.

Figure S1 shows the NW diameter as a function of growth time for two V/III ratios. As can be seen in Figure S1 (b), the diameter of smallest NWs increases with time as discussed in the main text. 


\section{Cooling down under $\mathrm{AsH}_{3}$ protection instead of $\mathrm{PH}_{3}$}

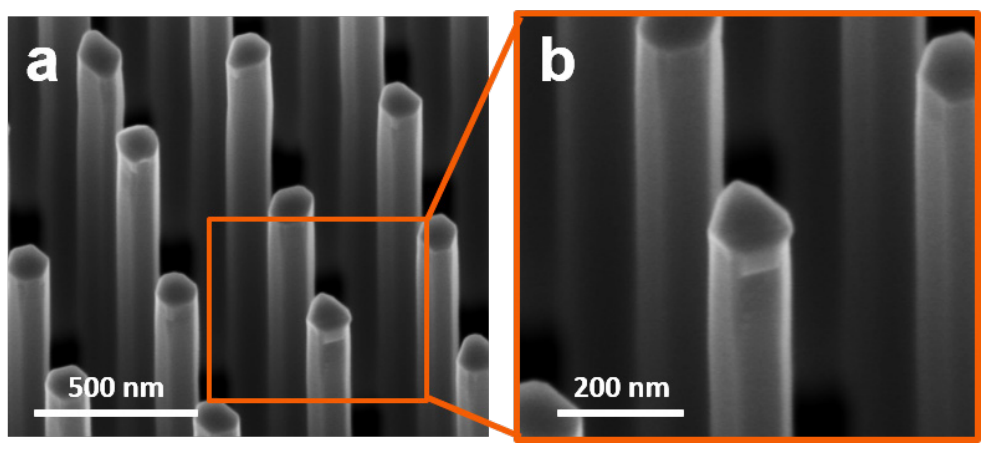

Figure S2. Scanning electron microscope (SEM) images of InP NWs cooled down under $\mathrm{AsH}_{3}$. (b) is a zoom-in image from the area highlighted in (a).

As can be seen in Figure S2, there are some visible features at the top of the NWs. The energy dispersive X-ray spectroscopy (EDX) results show that the top segment is As-rich, as shown in the main manuscript. 


\section{Nanowires grown on substrates patterned by FIB}
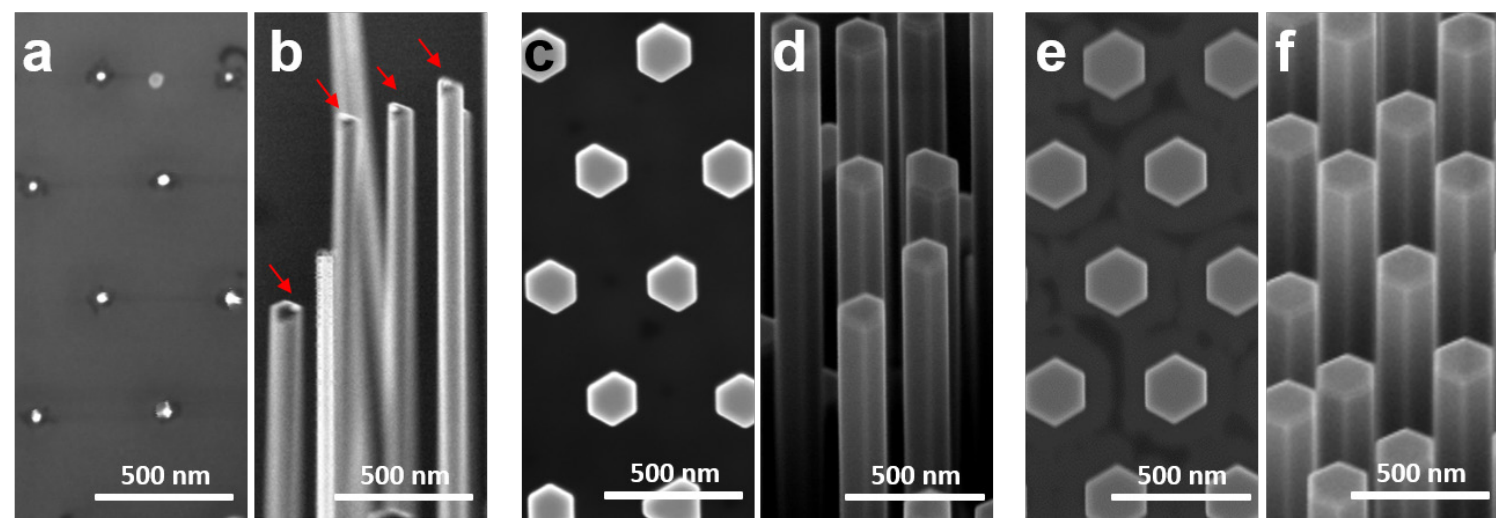

Figure S3. (a), (c), (e) and (b), (d), (f) are the SEM images at top and $30^{\circ}$ tilt view of InP

NWs grown on substrates patterned by FIB, respectively. The diameters of the hole openings are (a-b) 40, (c-d) 160 and (e-f) $220 \mathrm{~nm}$, respectively. It has been found that all NWs grown at a given opening size show some significant radial growth, explaining the difference between NW diameter and hole diameter. The red arrows in (b) indicate the positions of droplets.

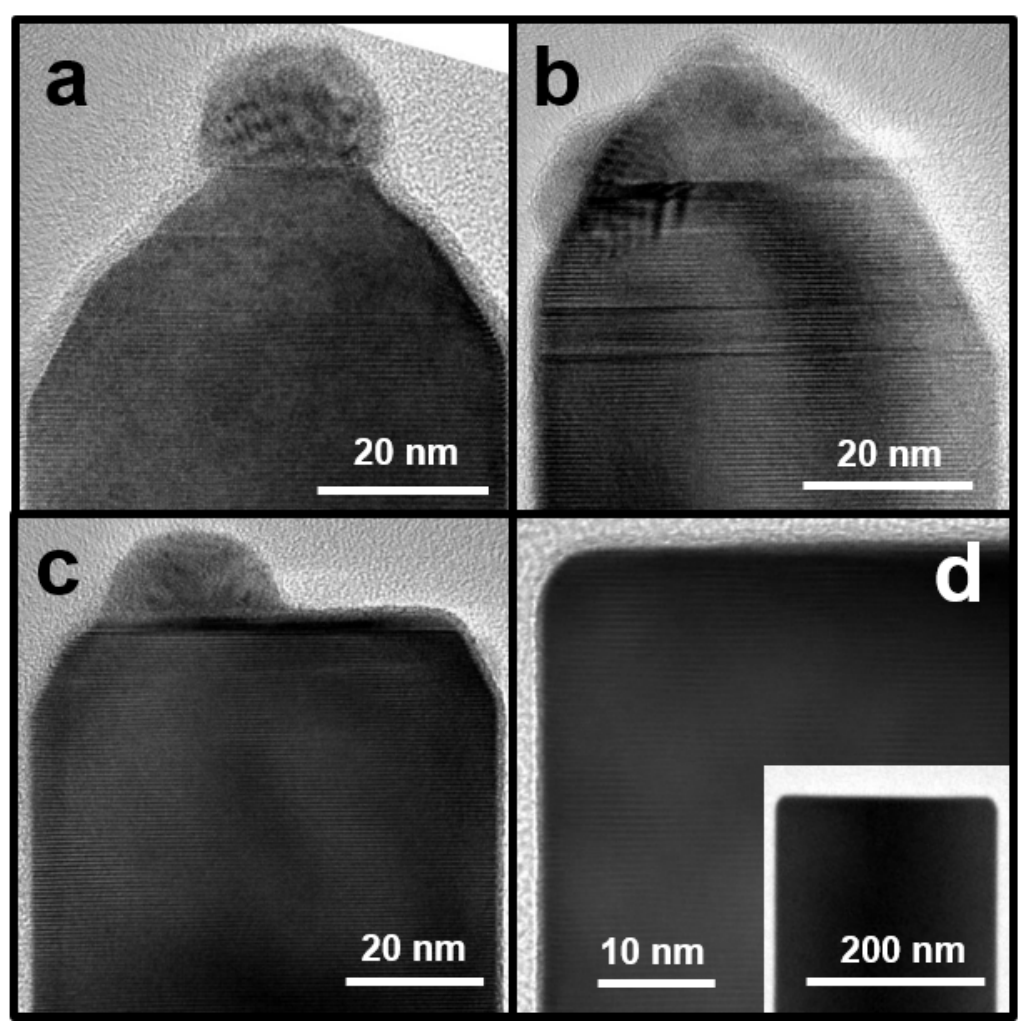


Figure S4. High-resolution transmission electron microscopy (HRTEM) images taken along [-2110] zone axis showing the morphology of the top of InP NWs with diameters of (a) 54, (b) 56 (c) 70 and (d) $239 \mathrm{~nm}$, respectively. There are indium droplets on the top of the NWs in (a-c). No droplet, stacking-fault or zinc blende segment can be found in the top of the NWs in $(d)$.

Figure S3 shows the scanning electron microscope (SEM) images of the InP NWs grown on substrates patterned by FIB. As can been seen from Figure S3 (b), small particles are formed at the top of the NWs while NWs with larger diameter have a flat surface without particles.

Figure S4 (a-d) shows the high-resolution transmission electron microscopy (HRTEM) images of the top of the InP NWs with different diameters. There are indium droplets on the top of the NWs in Figure S4 (a-c). The droplet in Figure S4 (b) has moved to the side and is visible in the top left corner of the TEM image. 


\section{Spacing study}

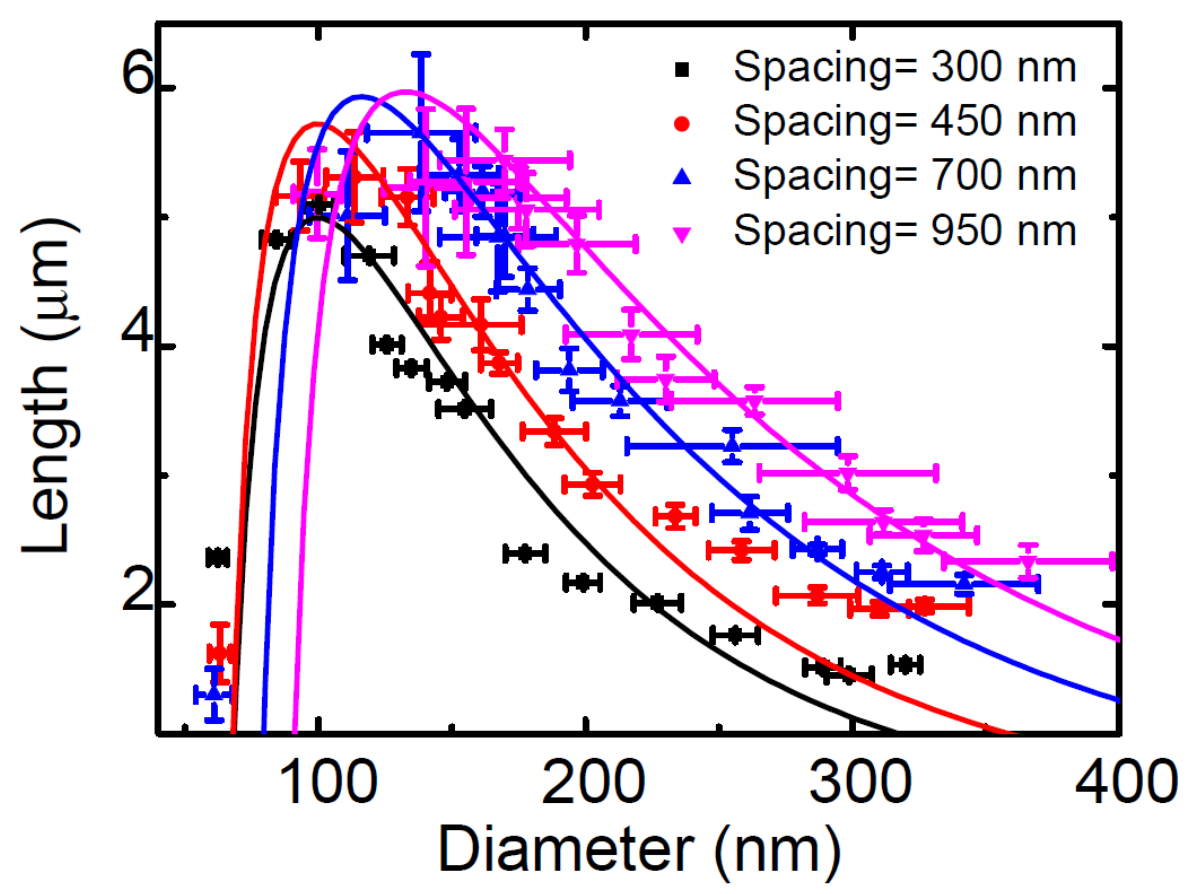

Figure S5. Graph of NW length as a function of opening diameter for four different pitches.

Lines show the fits by Equation (8) from the main text with the collection radius $R_{0}=620$, 710,910 and $1090 \mathrm{~nm}$ for the 300, 450, 700 and $900 \mathrm{~nm}$ spacings, respectively.

Figure S5 shows NW length as a function of opening diameter for the pitches of 300,450 , 700 and $950 \mathrm{~nm}$, respectively. The trend of these data is similar for different spacings with just a minor horizontal shift. For the same opening, NWs grown with larger spacing are longer (and have larger diameter) due to a larger collection area for growth precursors to contribute to the NW growth. These dependences are reasonably fitted by Equation (8) from the main text with $\lambda_{3}=2800 \mathrm{~nm}$ and the collection radius ranging from $620 \mathrm{~nm}$ for the smallest to $1090 \mathrm{~nm}$ to the largest spacing, as shown by lines in Figure S5. 


\section{Model for diffusion flux and nanowire length}

The diffusion flux of indium adatoms to the top of the NW is obtained by solving the stationary equation for the surface concentration of the sidewall gallium adatoms $n_{3}$

$$
D_{3} \frac{d^{2} n_{3}}{d z^{2}}+I_{3}-\frac{n_{3}}{\tau_{3}}=0
$$

with $I_{3}$ as the atomic influx of gallium, $D_{3}$ as the diffusion coefficient, $\tau_{3}$ as the effective lifetime of gallium atoms before desorption and $z$ as the vertical coordinate along the NW axis. The boundary conditions are taken in the form ${ }^{2}$

$$
-\left.2 \pi R D_{3} \frac{d n_{3}}{d z}\right|_{z=0}=I_{3} \pi\left(R_{0}^{2}-R^{2}\right), n_{3}(z=L)=0 .
$$

The first boundary condition implies that the diffusion flux to the NW base of radius $R$ equals the number of gallium atoms from the feeding area of radius $R_{0}$, and the second means that the adatom concentration is zero at the top of the NW. The solution is given by

$$
n(z)=I_{3} \tau_{3}\left[1-\frac{\cosh \left(z / \lambda_{3}\right)}{\cosh \left(L / \lambda_{3}\right)}\right]+\frac{\lambda_{3}}{D_{3}} \frac{I\left(R_{0}^{2}-R^{2}\right)}{R} \frac{\sinh [(L-z) / \lambda]}{\cosh (L / \lambda)},
$$

where $\lambda_{3}=\sqrt{D_{3} \tau_{3}}$ is the gallium diffusion length limited by desorption.

Diffusion-induced contribution to the NW elongation rate is obtained as

$$
v_{d i f f}=-\left.\frac{2 D \Omega_{35}}{R} \frac{d n_{3}}{d z}\right|_{z=L}
$$

where $\Omega_{35}$ is the volume per InAs pair in solid. Using Equation (S3) and introducing the gallium vapor flux $v_{3}=I_{3} \Omega_{35}$ and dimensionless diffusion flux $g_{\text {diff }}=v_{\text {diff }} / v_{3}$, we obtain 
Eqation (7) of the main text. The total NW elongation rate equals the diffusion flux plus the vapor flux, i.e. $d L / d t=v_{3}\left(g_{\text {diff }}+1\right)$. In the case $g_{\text {diff }}>>1$, we can write approximately

$$
\frac{1}{v_{3}} \frac{d L}{d t} \cong \frac{2 \lambda_{3}}{R} \tanh \left(\frac{L}{\lambda_{3}}\right)+\left(\frac{R_{0}}{R}\right)^{2} \frac{1}{\cosh \left(L / \lambda_{3}\right)} .
$$

Integrating this with the initial condition $L\left(t=t_{0}\right)=0$ yields Equation (8) of the main text with $\alpha=1$. The same procedure is used for obtaining Equation (8) from Equation (5) for arbitrary $\alpha$.

\section{REFERENCES:}

1. Gao, Q.; Saxena, D.; Wang, F.; Fu, L.; Mokkapati, S.; Guo, Y.; Li, L.; Wong-Leung, J.; Caroff, P.; Tan, H. H.; Jagadish, C. Nano Letters 2014, 14, (9), 5206-5211.

2. Dubrovskii, V. G.; Timofeeva, M. A.; Kelrich, A.; Ritter, D. Journal of Crystal Growth 2015, 413, 25. 